\title{
Design and Analysis of Transmitter Charge Switch Assembly Load on Weather Radar Test Platform
}

\author{
Shi Zheng1, Guan Wang2 ${ }^{*}$, Xingyou Huang ${ }^{3,4}$, Guilin Liu ${ }^{5}$ \\ ${ }^{1}$ Liaoning Provincial Meteorological Equipment Support Center, Shenyang, China \\ ${ }^{2}$ Liaoning Branch of Training School of Meteorological Cadres in China Meteorological Administration, Shenyang, China \\ ${ }^{3}$ Collaborative Innovation Center on Forecast and Evaluation of Meteorological Disaster, Nanjing University of Information \\ Science and Technology, Nanjing, China \\ ${ }^{4}$ Key Laboratory for Aerosol-Cloud-Precipitation of China Meteorological Administration, Nanjing University of Information \\ Science and Technology, Nanjing, China \\ ${ }^{5}$ Nanjing No. 14 Institutes, Nanjing, China \\ Email: 510042894@qq.com, *carfield53222@live.cn
}

How to cite this paper: Zheng, S., Wang, G., Huang, X.Y. and Liu, G.L. (2018) Design and Analysis of Transmitter Charge Switch Assembly Load on Weather Radar Test Platform. Journal of Geoscience and Environment Protection, 6, 51-58.

https://doi.org/10.4236/gep.2018.610004

Received: September 20, 2018

Accepted: October 28, 2018

Published: October 31, 2018

Copyright $\odot 2018$ by authors and Scientific Research Publishing Inc. This work is licensed under the Creative Commons Attribution International License (CC BY 4.0).

http://creativecommons.org/licenses/by/4.0/

\begin{abstract}
This paper provides a new method for designing the load of transmitters switch components on provincial radar test platform. Based on the calculation of the specific parameters related to the actual charge switch components, the load-related data model is obtained, the simulation is carried out, and the results are consistent with the actual test results. Through the theoretical numerical analysis of the load of the charging switch components of the transmitter device in the new generation of weather radar maintenance test platform, radar maintenance personnel at all levels can deepen the thorough understanding of the CINRAD/SA transmitter and have some enlightenment and improvement on the maintenance guarantee ability of the new generation of weather radar.
\end{abstract}

\section{Keywords}

Weather Radar, Charging Switch Assembly, Charging Equivalent Load, Charging Transformer

\section{Introduction}

In recent years, the new generation weather radar maintenance and maintenance test platform project have been completed and planned by the China Meteorological Administration and the meteorological bureaus of Liaoning, Inner Mon- 
golia, Shanghai, Hubei, Guangdong, Sichuan, Yunnan, Gansu and Xinjiang provinces, autonomous regions and municipalities in China. In order to maximize the functional benefits of the new generation weather radar maintenance and repair test platform, and to improve the maintenance and maintenance capabilities of radar support personnel, technicians at all levels of radar stations are required to fully understand the structural principles of the platform and understand the transmitter charging switch components. Load to design analysis ideas are crucial to future maintenance and repair work. The radar test platform is an open system, which is implemented by software radio technology. It can not only test existing radar equipment, but also add software modules to test different types of electronic equipment in different frequency bands. The provincial radar test platform supports the secondary development of the platform by the user, and can also be used as a radar training device for technical training.

Transmitter (SA) tool is the most important component of the new generation weather radar maintenance and maintenance test platform. It is extremely important to the radar test platform and is an important reference indicator for measuring the operation quality of the radar evaluation platform. When the radar test platform is working, the transmitter has high voltage, high current, high fault, and the charging switch component is prominent. How to detect whether the charging switch component works normally and whether the charging current waveform of the platform test switch component is satisfied (sawtooth wave) are the technical difficulties of test platform. Therefore, it is necessary to simulate and analyze the input and output circuits of the charging component, fully simulate the test signal of the component, the output equivalent load of the component, and further miniaturize the test tool, and finally reach the test requirement through simulation simulation design analysis [1]-[6].

The innovation of this paper is to test the existing weather radar equipment with a radar test platform, an open system. Taking Liaoning provincial radar maintenance test platform as an example, the input and output circuits of the charging component are simulated and analyzed. For the meteorological department, it is necessary to solve the problems faced by the technical support of the new generation weather radar equipment as soon as possible and improve the maintenance support capability.

\section{System Architecture of Provincial Weather Radar Test Platform}

Taking the China Liaoning Provincial weather radar maintenance test platform as an example, the Liaoning provincial radar test platform is based on the national test platform. At the same time, combined with the objective characteristics of the existing radar system construction in Liaoning Province, a fixed test platform is built, mainly including fixed test platform tool, fixed test platform instruments and software. The fixed test platform consists of instrumentation and special equipment, test tool, maintenance and test platform software. The 
fixed test platform tool mainly adopts off-line test and maintenance. The fixed software is mainly divided into six functional modules: basic database software, test platform software, TPS programs to test set, fault diagnosis program test set, expert experience library, remote test diagnosis software, instrumentation control and display assembly. The basic database software, test platform software, TPS program tests set are the basic functions, the fault diagnosis program test set, the expert experience library, and the remote test diagnosis software are comprehensively programmed and counted according to the number of tool components of the test component. The instrumentation control and display assembly is tested according to the number of instruments selected. The total system of Liaoning provincial radar test platform is mainly divided into five subsystems: the whole life cycle management subsystem of the tested component, the remote fault diagnosis subsystem, the expert experience library subsystem, the system integration and test subsystem and the system management subsystem. The system is designed in a "layered mode" with five levels: human-computer interaction layer, functional module layer, data layer, device driver layer and physical device layer. The working process of Liaoning provincial radar test platform is mainly divided into test analysis process and fault diagnosis process. The test analysis process is divided into a test modeling process and a test analysis process. The troubleshooting process is divided into a fault modeling process and a fault diagnosis analysis process.

\section{Design of Transmitter Charge Switch Component Load for Provincial Weather Radar Test Platform}

\subsection{Charging Switch Assembly Input and Output Circuit}

The SA transmitter three-phase rectifier component (A2) is filtered by the capacitor component (A9) and outputs DC510V as the DC power source of the charging switch component. The charging switch assembly charges and charges the charging transformer when the charging trigger timing comes. Since the charging transformer secondary is a different name termination method, the modulator artificial line is charged after the energy storage ends. When the discharge pulse comes, the voltage on the artificial line is controlled by the thyristor to discharge as shown in Figure 1.

Since the charging transformer is an inductive load, it can be known from the three elements of the circuit that the charging current is charged to a defined exponential relationship of a time constant $\tau(\mathrm{L} / \mathrm{R})$, and when $\mathrm{R}$ is small, it is approximately linear. The actual charging current waveform is shown in Figure 2. Due to the influence of the distributed capacitance of the charging transformer and the RC absorbing circuit of the modulator, the charging current starts with a very high current spike.

\subsection{Charging Switch Component Related Circuit Parameters}

Calculated from the operating parameters of the transmitter, the actual charging 


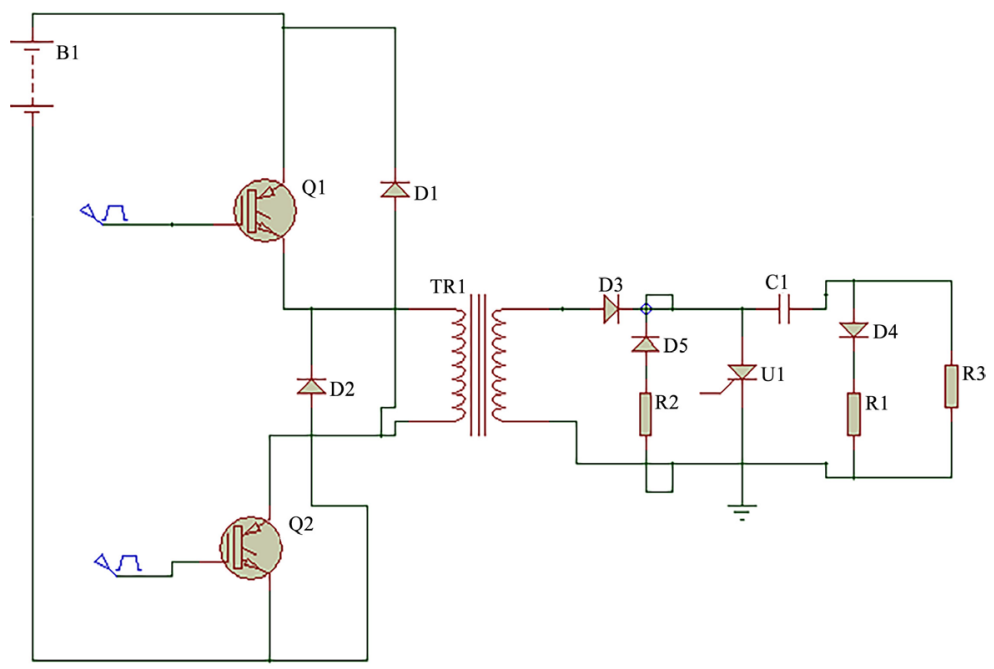

Figure 1. Simplified diagram of the actual charging switch assembly circuit (from Liaoning Provincial Meteorological Equipment Support Center).

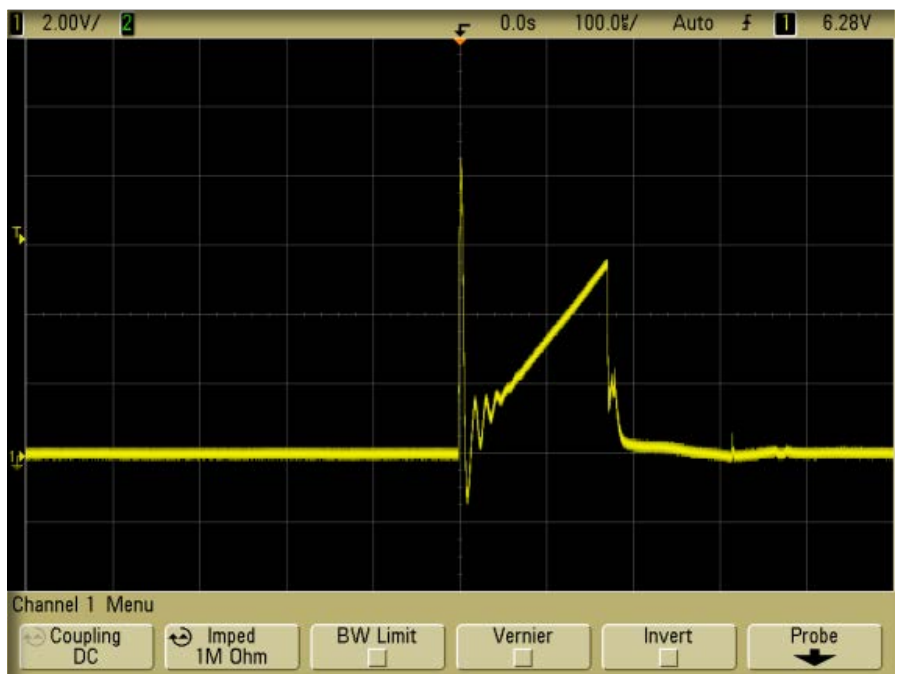

Figure 2. Actual charging current waveform.

time of the narrow pulse of the charging switch assembly is approximately $\mathrm{T}=$ $170 \mu \mathrm{S}$, the characteristic impedance of the artificial line of the modulator are 3 $\Omega$, and the pulse width of the modulator is designed to be $2.5 \mu \mathrm{S}$.

Then manual line capacitance is

$$
C_{P F N}=\frac{1}{2} \times \frac{\tau}{\rho}=\frac{1}{2} \times \frac{2.5 \times 10^{-6}}{3} \approx 0.417 \mathrm{uF},
$$

where $C_{P F N}$ is the manual line capacitance, $\tau$ is the pulse width of the modulator and $\rho$ is the characteristic impedance.

The energy stored by the artificial line is approximately equal to the energy converted by the inductor of the charging transformer, i.e.,

$$
W=\frac{1}{2} \times L, I^{2}=\frac{1}{2} \times C, U^{2}
$$


where $W$ represents work, $L$ represents inductance, $I$ represents current, $C$ represents capacitance and $U$ represents voltage.

Considering the charging efficiency $(\eta=0.9)$ and the artificial line voltage of $4600 \mathrm{~V}$, the equivalent inductance of the charging transformer is

$$
L=\frac{U_{D C}^{2} \times \eta \times T_{1}^{2}}{C_{0} \times U_{\max }^{2}}=\frac{510^{2} \times 0.9 \times\left(170 \times 10^{-6}\right)^{2}}{4600^{2} \times 0.417 \times 10^{-6}} \approx 0.767 \mathrm{mH},
$$

where $L$ represents the inductance, $U$ represents the voltage, $\eta$ represents the charging efficiency, $T$ represents the charging time, and $C_{0}$ represents the artificial capacitance.

According to the calculation of the three elements of the circuit, the peak value of the narrow pulse charging current is approximately (narrow pulse)

$$
I_{\text {Charging }}=\frac{U}{L} \times T=510 / L 1 * 170 \times 10^{-6} \approx 113 \mathrm{~A},
$$

and for wide pulses,

$$
I_{\text {Charging }}=\frac{U}{L} \times T=510 / L 1 * 300 \times 10^{-6} \approx 200 \mathrm{~A},
$$

where the symbolic meaning is the same as before.

From the above calculation, it can be concluded that the equivalent inductance of the charging transformer relative to the charging switch assembly are about $0.767 \mathrm{mH}$, the narrow pulse current is approximately $110 \mathrm{~A}$, and the wide pulse are $200 \mathrm{~A} \mathrm{[7]} \mathrm{[8].}$

\subsection{Calculation of Charge Load Switch Component's Equivalent Load}

Since the charging switch assembly accumulates a lot of energy on the inductor when it is storing energy to the charging transformer, the energy must be released before the next charging cycle. Therefore, a unidirectional circuit component is added to the equivalent load circuit. In summary, the equivalent circuit of the equivalent load of the switch component are shown in Figure 3. When the load is charged, due to the addition of a diode, its unidirectionality determines the energy stored in the inductor. When the energy is released after the end of the charging cycle, it is consumed by the diode on the high power resistor.

It is important to choose the right resistor and diode. Due to the high peak charging current, high power resistors and surface contact diodes must be selected. A suitable resistor size is also important. The resistance is too large, the energy release time is long, and the current has a reverse feedback phenomenon, and the true charging current waveform cannot be simulated. After simulation by the PROTUES circuit, it can be seen that the $100 \Omega$ resistor is used, and the energy is basically released after $50 \mu \mathrm{S}$. The current waveform of the actual provincial radar test platform charging switch component are shown in Figure 4, and the simulation waveform is basically the same as shown in Figure 5. 


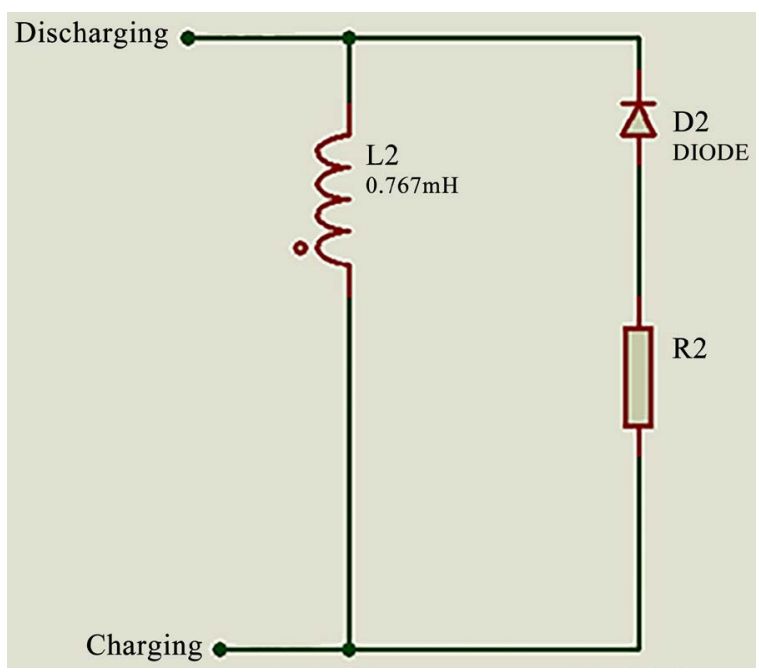

Figure 3. Charge switch assembly load equivalent circuit.

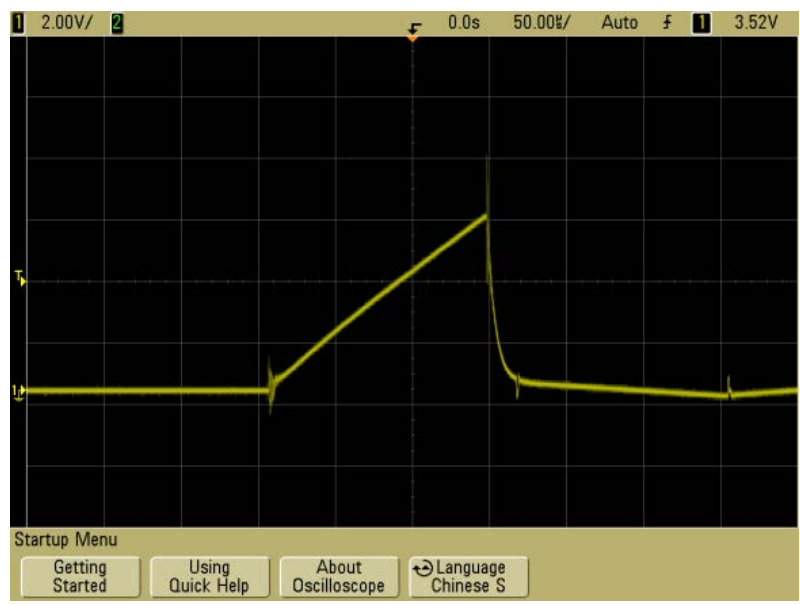

Figure 4. Test platform charging switch assembly actual test waveform.

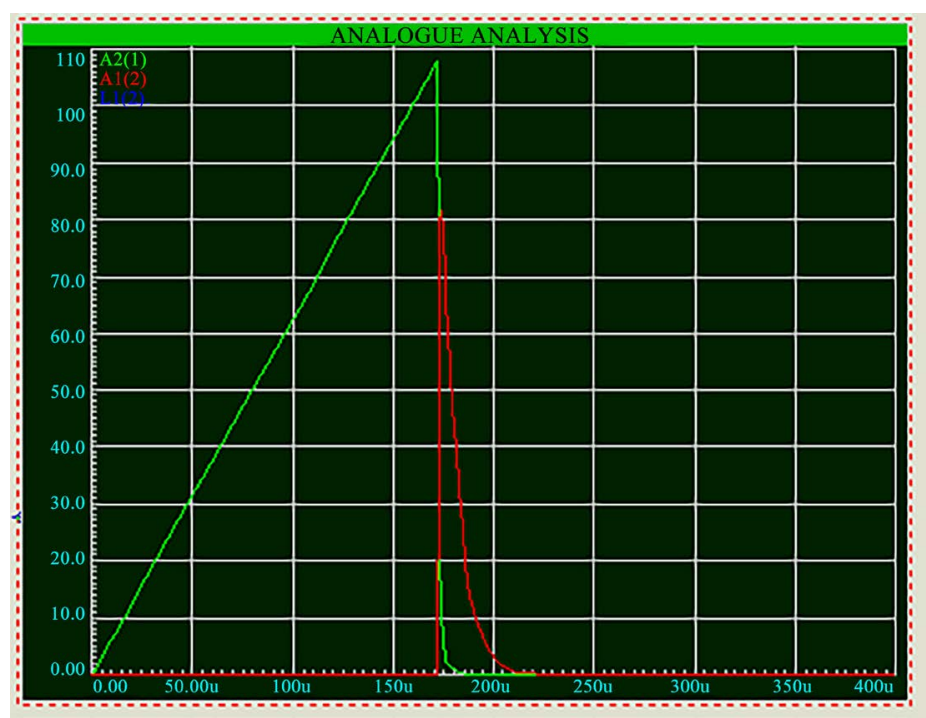

Figure 5. Charging component equivalent circuit simulation waveform. 
Compare with Figure 2, due to the distributed capacitance of the charging transformer and the RC snubber circuit of the modulator, the initial phase of the charging current has a very high current spike in Figure 2, however, in Figure 4, the charging switch component load is made by air core inductor, the distributed capacitance is small and there is no modulator RC circuit, and the current waveform has no actual current spike [9] [10].

\section{Conclusion and Discussion}

At present, most of the faults of the new generation of weather radars in China are basically solved by the manufacturers, but it is difficult to maintain the radar maintenance methods based solely on marketization. Market behavior has led to an increase in the cost of radar maintenance. As manufacturers use replacement components to eliminate radar failures, the number of technicians is limited, and the number and types of weather radars are numerous. As the use of new generation weather radars increase, the radar failure rate will increase year by year. High and security costs will also increase year by year, and the availability of radar services will inevitably decline year by year. In addition, after the completion of the new generation weather radar, it is difficult for manufacturers to maintain the existing technical personnel, which makes the protection more difficult. The meteorological department must solve the problems faced by the technical support of the new generation weather radar equipment as soon as possible. Therefore, it is necessary for the provincial meteorological departments in China to equip this weather radar test and maintenance system. After the system is completed, it can promote the construction of meteorological radar supports team, improve the technical level of radar maintenance personnel, reduce dependence on radar manufacturers, reduce radar maintenance and repair costs, and quickly respond to shorten radar maintenance time to ensure long-term stable and reliable operation of radar.

Some of the provincial radar test platforms that have been completed provide a wealth of text and graphic information such as connection diagrams, physical maps, functional block diagrams, and test procedures of the radar components to be tested. In order to better use the provincial radar test platform and improve the timeliness of the radar maintenance support work, each radar maintenance support personnel must thoroughly understand the principle and design ideas of the main software and hardware components of the platform, and can independently complete the platform inspection work. It also creates a radar test database that collects and intelligently analyzes, tests and diagnoses data, automatically generates test reports, and provides various forms of presentations. In the end, it will reduce the radar troubleshooting time, speed up the response, and improve the maintenance support capability.

\section{Acknowledgements}

This research was jointly funded by the China National High Technology Re- 
search and Development Program 863 Project (2007AA061901), the National Natural Science Foundation of China (40775029) and the Liaoning Provincial Radar Maintenance and Maintenance Platform Project, China.

\section{Conflicts of Interest}

The authors declare no conflicts of interest regarding the publication of this paper.

\section{References}

[1] Yang, J.H., Gao, Y.C. and Chai, X.M. (2014) Analysis of Operational Support Capability of New Generation Weather Radar. Meteorological Science and Technology, 42, 31-37.

[2] Liang, H., Gao, Y.C., Chai, X.M. and Liu, Y.Q. (2013) Typical Fault Analysis and Processing of CINRAD/CC Receiving System for New Generation Weather Radar. Meteorological Science and Technology, 41, 832-836.

[3] Liang, H., Chai, X.M. and Liu, Y.Q. (2013) Abnormal Diagnosis of Characteristic Curve of CINRAD/CC Radar Receiver. Meteorological Science and Technology, 41, 614-619.

[4] Yang, C.F., Chai, X.M., Zhang, H.Y., Huang, X.Z., Tu, A.Q., Liu, Z.H. and Yuan, X.Q. (2012) Technical Problems and Solutions for Digital Intermediate Frequency Transformation of CINRAD/SA Radar. Meteorological Science and Technology, 40, $5-8$

[5] Pan, X.M., Chai, X.M., Cui, Y.W., Xu, J.L., Huang, Y.Q. and Wang, Q.Z. (2011) Technical Features and Fault Diagnosis Methods of CINRAD/SB Radar Receiver. Meteorological Science and Technology, 39, 320-325.

[6] Pan, X.M., Chai, X.M., Cui, B.W., Huang, Y.Q. and Xu, J.L. (2011) Diagnostic Analysis of CINRAD/SB Radar Servo Power-On Fault. Meteorological Science and Technology, 39, 212-216.

[7] Tian, H. and Yuan, J.S. (2017) Analysis of Radar Communication Integrated System. Electronic Technology and Software Engineering, No. 23, 40.

[8] Zhao, L.J., Sun, X.D. and Yang, J. (2017) Radar Signal Processor Display Control and Communication Technology. Electronic Technology and Software Engineering, No. 15,47

[9] Song, W.J. (2017) Algorithm for Strong Pulse Interference Rejection of Continuous Wave Radar. Electronic Technology and Software Engineering, No. 15, 99.

[10] Cheng, H. (2017) Marine Radar Echo Simulation Based on DEM Image Enhancement. Computer Engineering and Applications, 1-5. 